\title{
Real-Time Microscopic Traffic Simulation and Optimization at Intersections with Video Traffic Detection
}

\author{
Zijun Liang, Hong Chen \\ College of Highway \\ Chang'an University \\ Xi'an, China \\ 953609319@qq.com, glch@chd.edu.cn
}

\author{
Yun-Pang Flötteröd, Alexander Sohr, Xiaoxu Bei, \\ Maximiliano Bottazzi, Jan Trumpold \\ Institute of Transportation Systems \\ German Aerospace Center (DLR) \\ Berlin, Germany \\ yun-pang.floetteroed@dlr.de, alexander.sohr@dlr.de, \\ xiaoxu.bei@dlr.de, maximiliano.bottazzi@dlr.de, \\ Jan.Trumpold@dlr.de
}

\begin{abstract}
- in this paper, real-time vehicular data from video traffic detection (VTD) are used for minimizing the travel delay at intersections and a real-time traffic optimization model, based on the SUMO traffic simulation software, is established accordingly. The proposed model is implemented in a small industrial control computer which serves as the communication interface between the traffic signal control system, the traffic simulation and optimization model and the real-time video traffic detection equipment to optimize the respective signal timing plans. The decision to shorten or extend the respective green time can be made at each second under the current development. To verify the results of the simulation study a field study is undertaken in Hefei, China. The overall intersection performances before and after the signal timing optimization are calculated and compared. The results show that the method proposed in this paper can significantly minimize the vehicle delay and the corresponding air pollution in real time.
\end{abstract}

real-time intersection optimization; online simulation; SUMO; VITAL

\section{INTRODUCTION}

Serious traffic congestions and the respective pollution problem have become one of the critical issues that worldwide governments have to face in metropolitan areas. Intersections are the critical locations that affect the efficiency of traffic operation. The efficiency of traffic signal operation has therefore become one of the important indicators to evaluate the performance of city traffic management. Diverse researches on traffic signal optimization have been undertaken. With the technology innovation more researches have focused on real-time signal control optimization [1-4]. Different traffic control systems have also been developed and applied in practice, such as the Australian SCATS system, the British SCOOT system and the German ACTRA system [5-6]. Moreover, microscopic traffic simulation has extensively applied for traffic forecasting and strategies evaluation in the traffic management field. The respective application trend has obviously shifted from offline to online [7]. For example, Lin, Liu and Yao [8] proposed a method to assist decision making with use of online traffic simulation. With this method the optimal emergency treatment plan can be promptly and quantitatively identified after a traffic incident occurred. Wu, Shi and Xie [9] proposed a real-time calibration method for online microscopic traffic simulation at intersections. In this method, platoon dispersion diagram is used as the fit indicator. The calibration works by constantly adjusting the selected parameters until the difference between the simulated and the real platoon dispersions is less than the pre-defined threshold. Zhang, Zheng and Li [10] carried out the synchronization between the simulation and the reality and reproduced the traffic situation in the investigated area. They proposed the interface components of traffic data and control data so that the microscopic traffic simulation VISSIM can be connected with the real traffic data. Furthermore, Deng and Li [11] proposed the datadriven intelligent traffic virtual simulation system architecture and the corresponding key technologies in a big data environment. Jia, Wu and Du [12] used FLOWSIM as an example to demonstrate the possible applications of microscopic traffic simulation in city traffic management.

The above mentioned researches have reached certain academic achievements. However, most of the proposed methods are still limited for practical application. Moreover, the advantage of open source traffic simulation software, e.g. flexibly adjust/develop customized interfaces and functions, has not been effectively utilized, especially for studying the traffic-related environmental issues at intersections in China. This study focuses therefore on the real-time optimization of traffic signals and the evaluation of the respective traffic emission productions with use of microscopic traffic simulation and the video traffic detection data. The corresponding implementation and the applicability in practice are also the focus in this paper. In the following, the used methodology and software are described in Section II. Section III explains the respective implementation work for practical application. After that, the field study area and the current traffic situation are illustrated in Section IV. Section $\mathrm{V}$ shows the results both from the field study and the simulation. Finally, the corresponding conclusions and the perspectives are given in Section VI. 


\section{METHODOLOGY}

The applied methods in the proposed model include the delay-based traffic signal optimization and the SUMO traffic simulation suite and are explained below.

\section{A. Delay-based Traffic Signal Optimization}

With the innovative development in Information and Communication Technologies (ICT) fine data from video capturing, wireless detectors and vehicle-to-infrastructure (V2X) communication become available in real time. Such data help to further model traffic phenomena in detail and optimize traffic control/management strategies effectively. Thus, the way to control traffic signals can be changed from traditional static control to demand responsive control for improving the operation efficiency of traffic signals.

The main concept of the developed delay-based control method, illustrated in Fig. 1, is to adjust the green time duration in each signal timing phase with regard to vehicles' delay times, which can be derived from the collected ICT data [13]. Given the signal timing phases, the maximum and minimum green times as boundaries a green time phase is terminated either when all vehicles with delays has been served on the respective approach or the maximum green time is reached. A vehicle is considered to have delays if its current speed on an approach is lower than the corresponding speed limit. Generally speaking, delay time includes initial deceleration time, queue move-up time, stopped time, and final acceleration time [14]. This method has been tested and validated with two German case studies in the VITAL project (Vehicle-Actuated Intelligent Traffic Signal Control) [15-16] and is therefore also called the VITAL method.

Due to the method characters, i.e. the phases and the respective sequence plan are fixed, the main improvement occurs during the off-peak periods. During the peak periods, there are high traffic volumes at intersections and the duration of the given maximum green time is often reached. Thus, the respective improvement is limited when comparing the traffic delay with that from the given static traffic signal control program [17].

\section{B. SUMO traffic simulation suite}

DLR's SUMO (Simulation of Urban Mobility) is applied for the real-time signal optimization and quantitatively analyzing the influence of the VITAL method both on the environment and the intersection performance in this study. SUMO is an open source, highly portable, microscopic and continuous road traffic simulation package and is designed to handle large road networks. It has been continuously developed for more than 15 years and has be extensively successfully applied in different projects related to urban traffic management, traffic emission, V2X and other diverse traffic issues [18-19]. Some simulation works [17, 20] with SUMO for the City Hefei, China has been conducted as well.

SUMO's emission model is based on the emission model HABEFA 3.1 (The Handbook of Emission Factors for Road Transport) [21]. The results in [17] show that the time series trend of the simulated emissions (CO, NOx) corresponds quite well with that of the respective measured immissions, especially during the daytime when most of traffic occurs. The non-vehicle emissions cannot be simulated by SUMO.

In order to collect the simulated emissions at the subject intersection SUMO's TraCI (Traffic Control Interface) is used so that the emission of each simulated object can be retrieved and collected during a running traffic simulation.

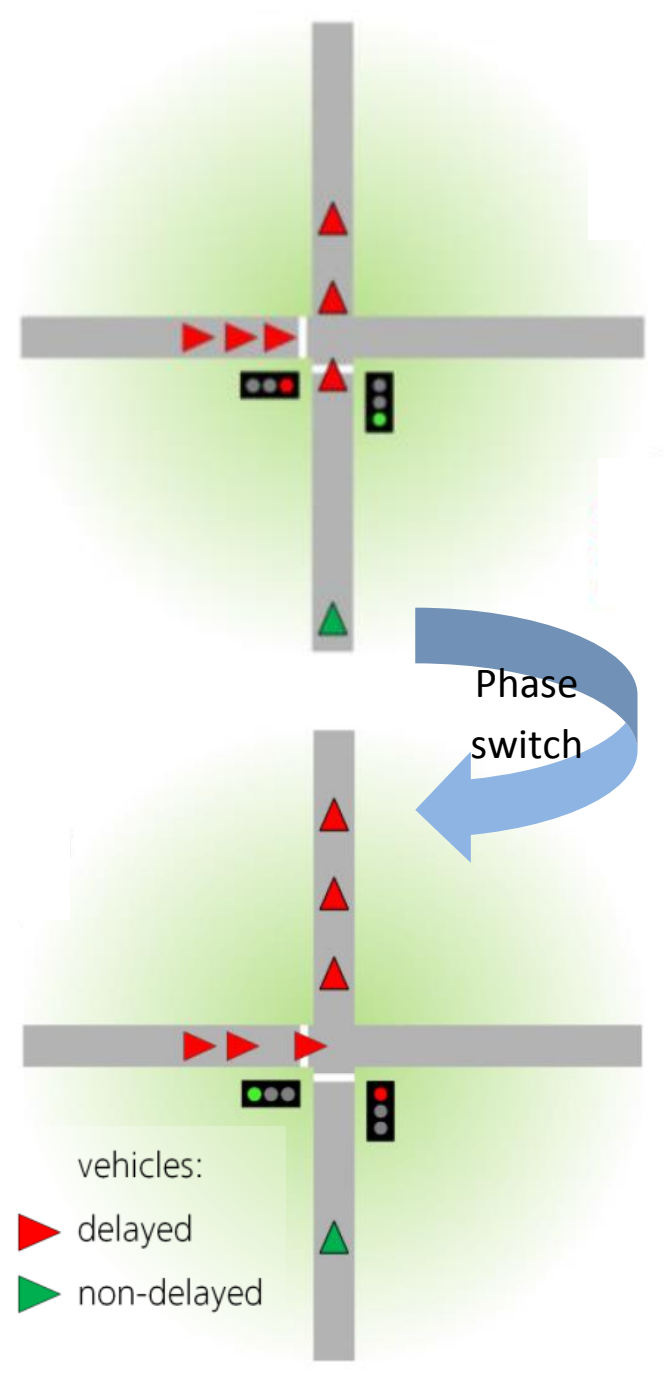

Figure 1. Concept of the delay-based method.

\section{SYSTEM ARCHITECTURE AND IMPLEMENTATION}

Instead of using loop detectors video traffic detection technique is used in this study. The collected data are sent wireless to the control center. Therefore, special attention has been paid to the network latency when implementing the system. Traffic safety issue is also taken care of regarding the signal phases and the vehicle clearance time.

\section{A. System Architecture Design and Working Principle}

The whole system consists of a small industrial control computer (IPC), an intersection signal controller and the 
video detectors at the intersection. All these components are connected by external physical interfaces. The IPC and the video detection server are deployed in the cabinet of the respective signal controller in order to shorten the communication distance and ensure that the communication chain is stable and reliable even under the harsh environment. The system architecture design is shown in Fig. 2. The applied VITAL method is implemented in SUMO. The IPC serves as the operating carrier of the SUMO-based traffic simulation and optimization model, and is connected to the signal controller and the video detector via the external interfaces RS232 and RS485 respectively.

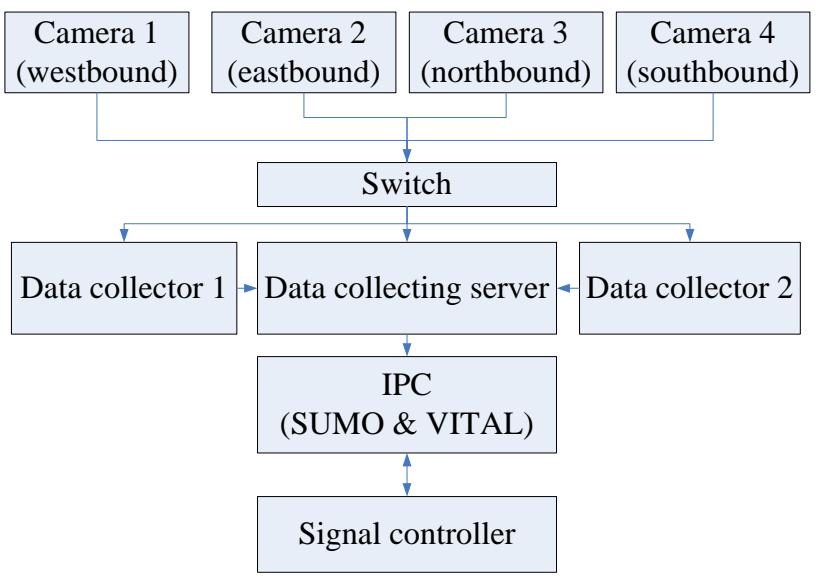

Figure 2. System architecture design.

The working process of the system is explained as follows. The videos made by the camera in each direction are sent to the data collectors. After that, the data collecting server sends the real-time traffic flow data to the IPC as the basic input data. If a video detector is occupied, a corresponding vehicle is generated in SUMO for traffic simulation and signal optimization. Moreover, the traffic signal controller sends the current phase state to the IPC and, almost at the same time, receives and executes the signal control command from the IPC, i.e. shorten or extend the respective green time. The phase sequence plan remains the same. Only the duration of the respective green time will be adjusted according to the traffic flow data from the video detectors. If the given maximum green time is reached or there is no delayed vehicles on the respective approach, IPC will send the phase switch command to the signal controller. The current green time phase will then be terminated and the next phase will be activated. All the actions sent by the IPC depend on the real-time intersection simulation and evaluation, executed by the embedded SUMO traffic simulation.

\section{B. Data communication protocol}

The way to connect the IPC and the traffic signal controller is to use a RS232 bus. The adopted communication baud rate is 57600 . The data consist of one header, eight digit bits and one end bit. No parity bit is applied here. The traffic signal controller and the IPC send data to each other every second to optimize the traffic control in real time. The data length is totally eight bytes and the respective definition is shown in Table I.

Furthermore, RS485 bus is used to connect the IPC and the video detectors for transmitting large data in real time. The respective communication baud rate is 115200 . The data include one header, eight digit bits and one end bit without any parity bit. The data format definition is indicated in Table II.

TABLE I. DATE TRANSMISSION FORMAT BETWEEN THE IPC AND THE SIGNAL CONTROLLER

\begin{tabular}{|c|c|c|}
\hline \multirow[b]{2}{*}{ Name } & \multicolumn{2}{|c|}{ Format Definition } \\
\hline & $\begin{array}{c}\text { from the signal controller } \\
\text { to the IPC }\end{array}$ & $\begin{array}{c}\text { from the IPC to the signal } \\
\text { controller }\end{array}$ \\
\hline $\begin{array}{l}\text { Header } \\
\text { (1 byte) }\end{array}$ & 0XE5 & 0XE5 \\
\hline $\begin{array}{l}\text { Signal work } \\
\text { mode } \\
\text { (1 byte) }\end{array}$ & 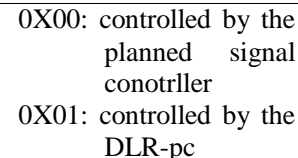 & - \\
\hline $\begin{array}{c}\text { Program } \\
\text { number } \\
\text { (1 byte) }\end{array}$ & $1-32$ & - \\
\hline $\begin{array}{l}\text { Phase number } \\
\text { (1 byte) }\end{array}$ & $1-32$ & - \\
\hline $\begin{array}{l}\text { Phase state } \\
(1 \text { byte })\end{array}$ & $\begin{array}{l}\text { 0X00: normal state } \\
\text { 0X01: transition state }\end{array}$ & - \\
\hline $\begin{array}{l}\text { Working state } \\
\text { (1 byte) }\end{array}$ & 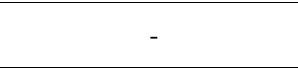 & $\begin{array}{l}\text { 0X00: abnormal } \\
\text { 0X01: normal }\end{array}$ \\
\hline $\begin{array}{l}\text { Switch state } \\
\text { (1 byte) }\end{array}$ & - & 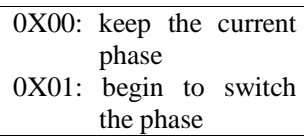 \\
\hline Reserved & reserved ( 2 bytes) & reserved ( 4 bytes) \\
\hline End (1 byte) & $0 \times 5 C$ & $0 \times 5 \mathrm{C}$ \\
\hline
\end{tabular}

TABLE II. DATE TRANSMISSION FORMAT BETWEEN THE IPC AND THE VIDEO TRAFFIC DETECTORS

\begin{tabular}{|l|c|c|}
\hline \multirow{2}{*}{ Name } & \multicolumn{2}{|c|}{ Format definition } \\
\cline { 2 - 3 } & $\begin{array}{c}\text { When a vehicle enters } \boldsymbol{a} \\
\text { detector }\end{array}$ & $\begin{array}{c}\text { When a vehicle leaves a } \\
\text { detector }\end{array}$ \\
\hline $\begin{array}{l}\text { Header } \\
(1 \text { byte })\end{array}$ & $0 \mathrm{XF0}$ & $0 \mathrm{XF1}$ \\
\hline $\begin{array}{l}\text { Detector } \\
\text { number } \\
(1 \text { byte })\end{array}$ & $1-39$ & $1-39$ \\
\hline $\begin{array}{l}\text { Occupancy } \\
\text { time (2 bytes })\end{array}$ & - & integer \\
\hline End (1 byte) & $0 X 5 \mathrm{C}$ & 0 0X5C \\
\hline
\end{tabular}

\section{FIELD STUDY}

To examine the proposed system and model mentioned in Sections II and III, the intersection at Huangshan Rd. and Tianzhi Rd. in Hefei, China is selected for the field study. This intersection has equipped different types of sensors and communication devices for different researches on ICTdevice testing and validation as well as on the intelligent 
traffic management. The collected data are also the important data source for understanding the local driving behaviors, such as car following and lane changing. In the following, the executed field study is explained in five parts: the current infrastructure, the applied video detection, the existing traffic situation, the designed traffic signal plans and the traffic simulation establishment.

\section{A. Infrastracture}

The subject intersection has equipped different types of sensors and communication devices for different researches on intelligent traffic management system. Huangshan Rd. is the main arterial with 4 lanes in each direction and serves the east- und westbound traffic. Tianzhi Rd. is the minor street with two lanes in each direction. The intersection layout and the sensor allocation are depicted in Fig. 3(a) and (b) respectively. The detectors, indicated with numbers in the squares are vehicle detectors, where 1-13 and 27-29 represent the detectors at the road sections and at the intersection respectively. The traffic signals, indicated with numbers from 1 to 15 in the circles are for vehicles and those with numbers from 21 to 24 in the ovals are for pedestrians.

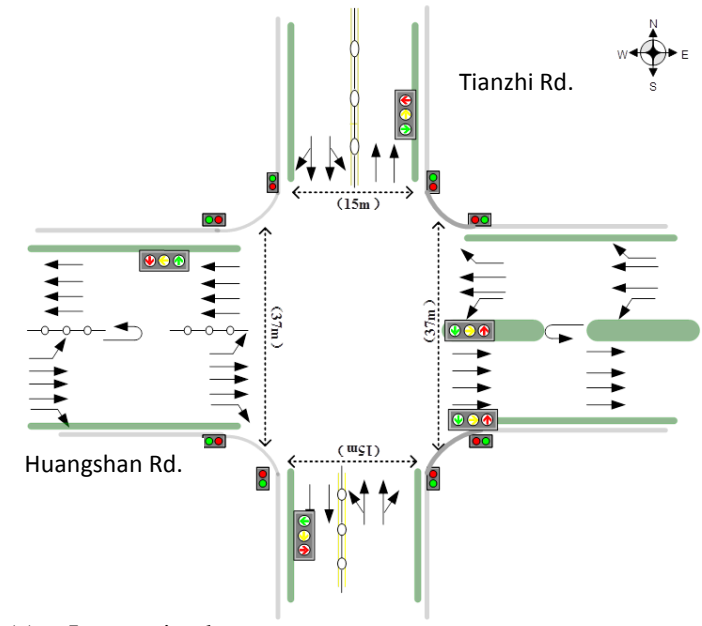

(a) Intersection layout

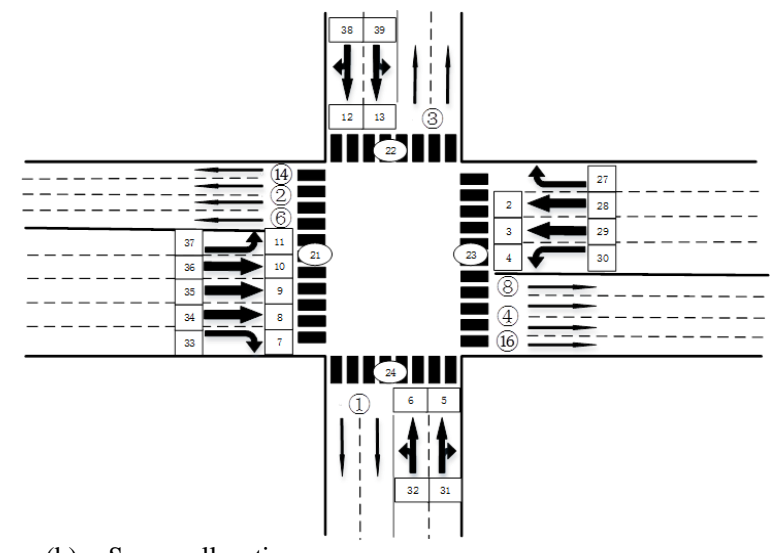

(b) Sensor allocation

Figure 3. Illustration of the infrastructure at the subject intersection.

\section{B. Viedo Traffic Detection}

To overcome the intensive computation of vehicle tracing and the issue that vehicles can be obscured by other subjects an improved Kalman filter detection algorithm is adopted in the video traffic detection process. Through the prediction of the characteristics of a vehicle, such as vehicle position and vehicle edge, the moving target can be found and then matched with the current frame for finding the corresponding relation in the image sequence and ensure the respective trajectories. When a vehicle is hidden, the matching template is updated with use of the vehicle body edges before and after the occlusion in order to improve the matching accuracy.

The experimental results showed that this method can accurately detect the moving objects in the given video images, and can also perform well when there are changes in the brightness and background of the scene in real time. Fig. 4 shows two screenshots of the applied video detectors.

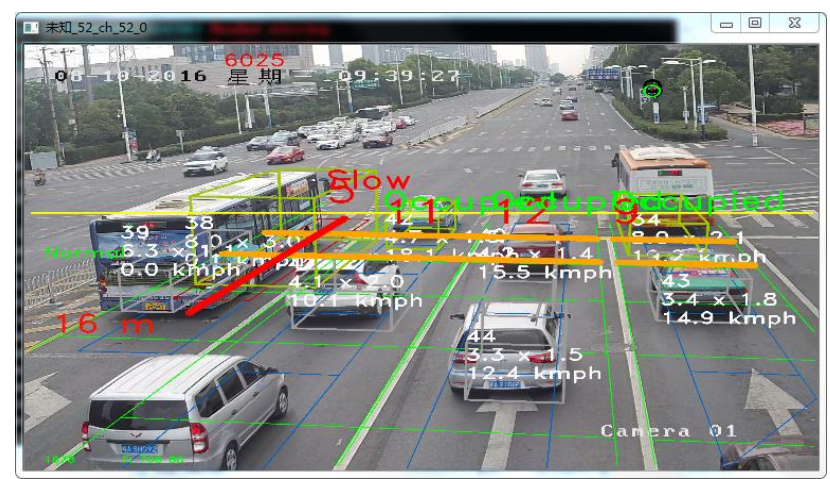

(a) at the subject intersection

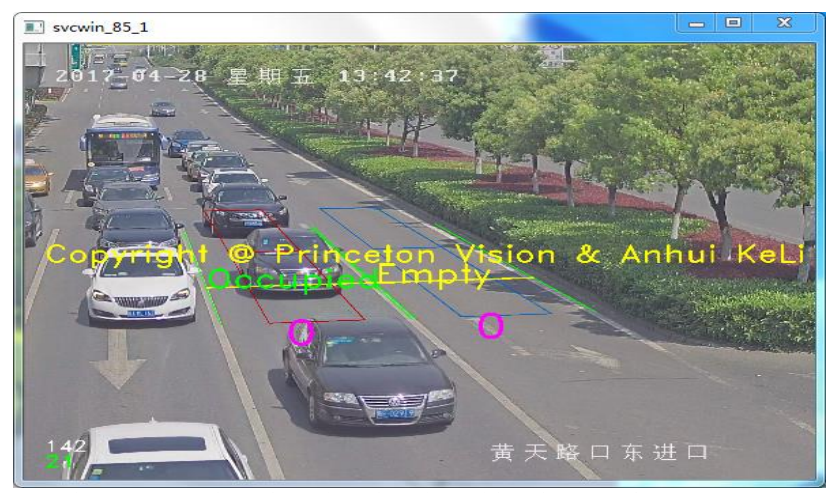

(b) at a road section closed to the subject intersection

Figure 4. Screenshots from the Deployed Video Detectors.

\section{Current Traffic Situation}

The traffic flow time series on a normal workday is illustrated in Fig. 5. The traffic is mainly passenger cars. Some buses also exist, but mainly during the peak-hour periods. Trucks and large delivery vehicles are not allowed during daytime. The morning and evening peak periods are between 7 and 9 o'clock and between 17 and 19 o'clock 
respectively. After 19 o'clock, the traffic in the eastbound direction is still quite high in comparison to that in the other directions.

Furthermore, the saturation headways and flows are collected and calculated respectively (see Table III). It is obvious that the saturation headways in the east- and westbound directions are less than those in the south- and northbound directions. It is mainly since the lane use groups are separated, i.e. each traffic movement has its own lane(s), and the signal phases are protected for each traffic movement. Due to the shared lanes and signal phases the south- and northbound traffic has larger headways to ensure the traffic safety. Such higher headways result in lower saturation flow rates, especially for the left-turn traffic.

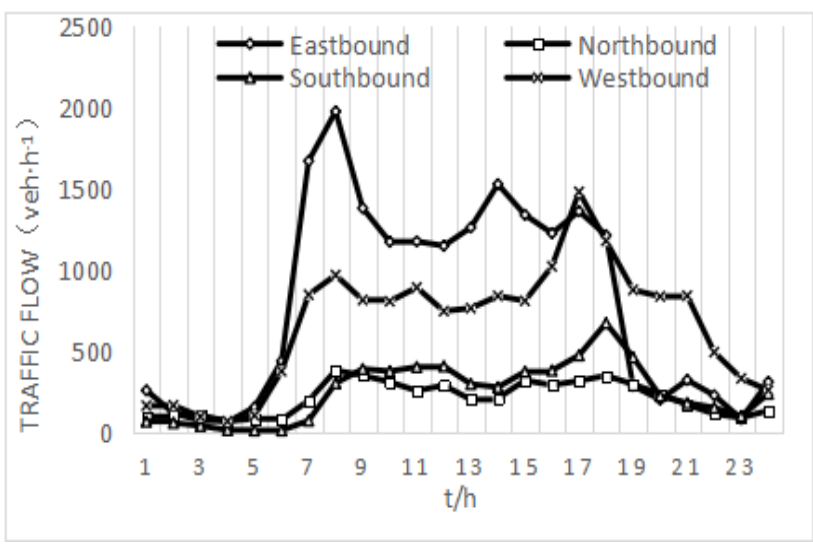

Figure 5. Traffic Flow Time Series on a Normal Workday.

TABLE III. SATURATION Flow RATE AT THE INTERSECTION

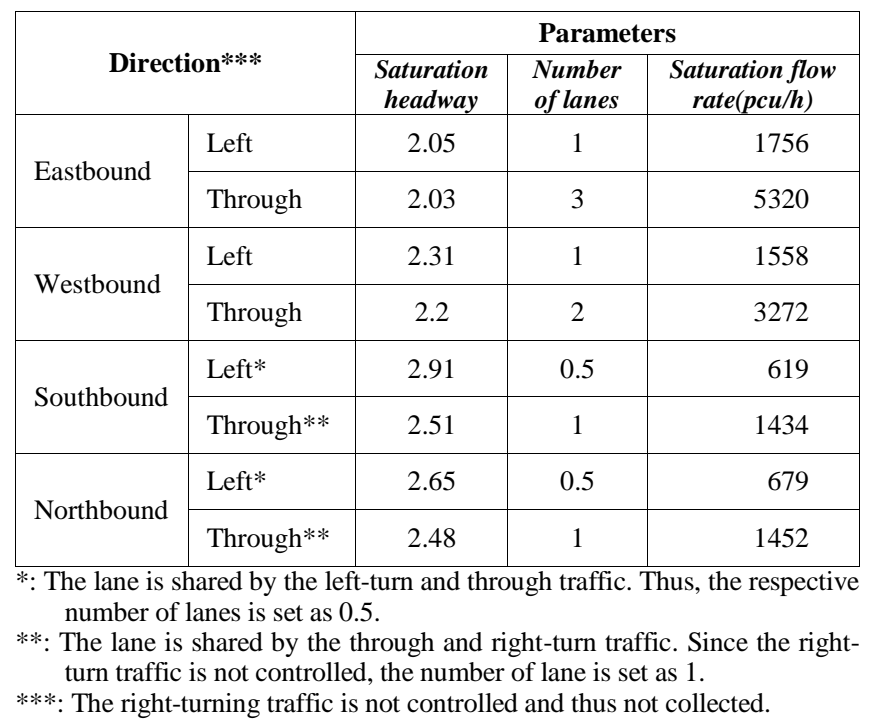

With the consideration of the characters of the VITALmethod, mentioned in Section II, the field test will focus on the off-peak period from 11 to 16 o'clock. Table IV shows the average traffic flows during the off-peak period. The through traffic is the main traffic in the east- and westbound directions, where $29 \%$ of the westbound traffic is left-turn traffic. It indicates that the current protected left-turn phase corresponds to the left-turn traffic demand. On the other hand, there are also higher left-turn ratios in the south- and northbound direction (65\% and 30\%). Due to the lower through traffic demand the left-turn traffic can find a proper gap to go through the intersection. Thus, there is no need to set a protected left-turn phase which also corresponds to the current signal program.

TABLE IV. AVERAGE HOURLY Flow RATE AT THE INTERSECTION DURING THE OFF-PEAK PERIOD (11-16 O'CLOCK)

\begin{tabular}{|c|c|c|c|c|}
\hline \multirow{2}{*}{\multicolumn{2}{|c|}{ Direction }} & \multicolumn{3}{|c|}{ Road Parameters } \\
\hline & & \multirow{2}{*}{$\begin{array}{c}\begin{array}{c}\text { Flow rate } \\
\text { (pcu/h) }\end{array} \\
30\end{array}$} & \multirow{2}{*}{$\begin{array}{c}\begin{array}{c}\text { Ratio/ } \\
\text { Movement }\end{array} \\
0.04\end{array}$} & $\begin{array}{c}\text { Ratio/ } \\
\text { Direction }\end{array}$ \\
\hline \multirow{2}{*}{ Eastbound } & Left & & & \multirow{2}{*}{0.48} \\
\hline & Through & 700 & 0.96 & \\
\hline \multirow{2}{*}{ Westbound } & Left & 330 & 0.29 & \multirow{2}{*}{0.31} \\
\hline & Through & 800 & 0.71 & \\
\hline \multirow{2}{*}{ Southbound } & Left & 130 & 0.65 & \multirow{2}{*}{0.12} \\
\hline & Through & 70 & 0.35 & \\
\hline \multirow{2}{*}{ Northbound } & Left & 80 & 0.29 & \multirow{2}{*}{0.09} \\
\hline & Through & 200 & 0.71 & \\
\hline
\end{tabular}

\section{Signal Timing Plans}

The current traffic signal program for the analysis period has three signal phases. The first and the second phase are for the west- and eastbound traffic respectively. The southand northbound traffic shares the third phase together. All right turning traffic is not controlled by the traffic signal. According to the observed traffic situations, the current signal phases correspond to the existing traffic demand. Thus, only the green time durations will be adjusted in the field study. The designed timing plans are shown in Table V.

TABLE V. PROPOSED GREEN TIME DURATIONS

\begin{tabular}{|l|c|c|}
\hline \multirow{2}{*}{\multicolumn{1}{|c|}{ Direction }} & \multicolumn{2}{c|}{ Green Time (sec) } \\
\cline { 2 - 3 } & Minimum duration & Maximum duration \\
\hline Eastbound & 10 & 31 \\
\hline Westbound & 12 & 37 \\
\hline South- and Northbound & 18 & 33 \\
\hline
\end{tabular}

\section{E. The traffic optimization and simulation model}

According to the traffic data, detector allocation, traffic signal phases and the designed signal timing plans the traffic simulation at the subject intersection is established. The simulation layout is shown in Fig. 6. The real-time data will be directly sent to SUMO and the VITAL-method for the 
traffic simulation and the traffic signal optimization. The simulated traffic emissions at the intersection with a radius of 90 meters are also collected for analyzing the environmental influence of the VITAL-method. Furthermore a gap-based actuated control method [22], which is often applied in Germany, is adopted as a reference control method. The main idea of the gap-based actuated control is to switch the signal phase after detecting a sufficient time gap between successive vehicles. This method aims at forming a better distribution of green-time among phases and affecting the respective cycle duration in response to dynamic traffic conditions.

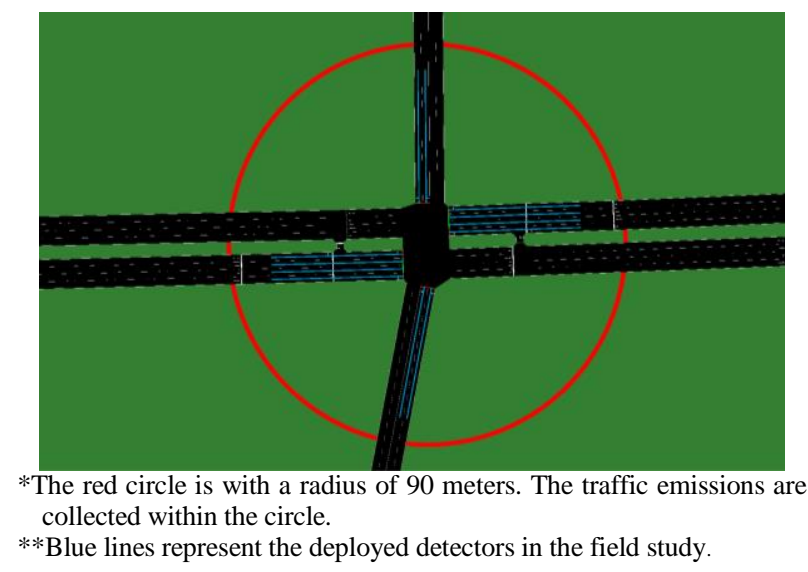

Figure 6. Layout of the simulative intersection.

\section{RESUlTS}

The simulation study is firstly carried out to compare the VITAL method with the current pre-timed and the gap-based actuated control methods. Both the intersection operation efficiency and the environmental impact are investigated. Furthermore, the average vehicular delay and the number of vehicular stops before and after the application of the VITAL method in the field study are compared for understanding the affect that the applied online traffic simulation and optimization model has brought in practice.

\section{A. Simulation results}

The collected data are limited in the field study due to some technical reason. The vehicle data collected in June 2017 are thus used in the analysis. Fig. 7 (a) shows the average delays per vehicle from 11 to 16 o'clock with three different traffic control methods. The actuated and the VITAL-method can both substantially reduce the delay time in comparison to the pre-timed method. The reduction ratio in the 6 hours is $45 \%$ and $57 \%$ respectively. The VITAL method can reduce more delay time than the actuated method, especially in the period (15-16 o'clock) close to the traffic peak period (17-19 o'clock). The similar result can also be found when observing the stopping duration (see Fig.
7 (b)). The average stopping duration keeps quite constantly, between 5 and 8 seconds, when applying the VITAL method. In comparison to that, the actuated method is more dependent on the amount of traffic and the stopping duration varies between 6 and 22 seconds.

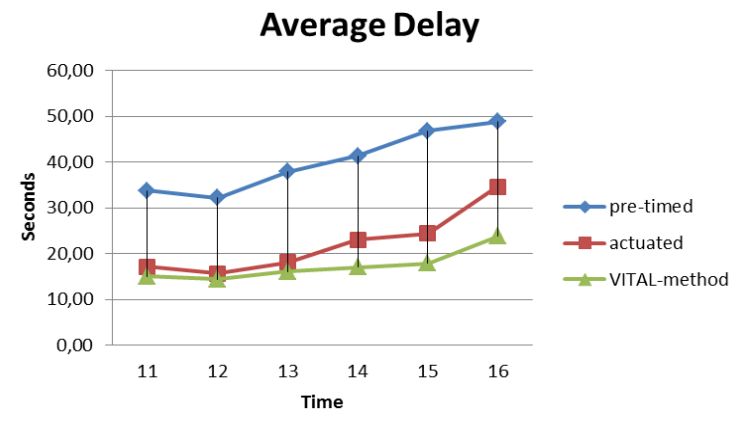

(a) Average delays of three different control methods

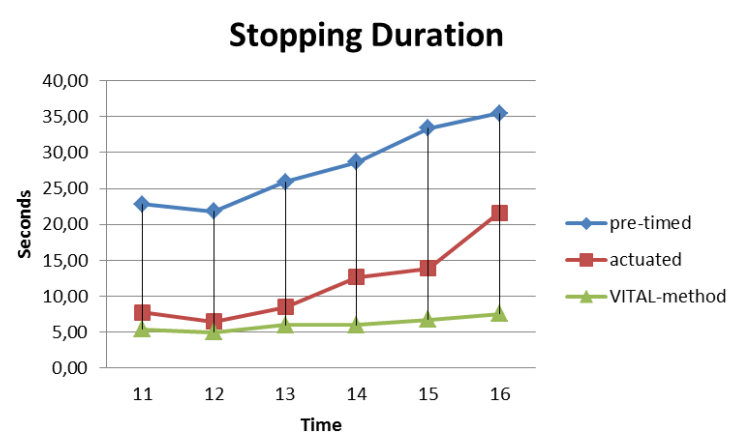

(b) Average waiting times of three different control methods

Figure 7. Comparion of the simulated intersection performance.

In regard to the environmental influence the VITAL method results in the least amount of emission productions among the three applied control method. Fig. 8 indicates that the applications of the VITAL method and the actuated method have a significant reduction in $\mathrm{CO}$ when comparing with the pre-timed method. The total reduction ratio in the overall observation period is $44 \%$ and $34 \%$ respectively. The reduction ratio in $\mathrm{NO}_{\mathrm{x}}$ is $22 \%$ with the actuated method and $24 \%$ with the VITAL method. The reduction ratio in $\mathrm{PM}_{\mathrm{x}}$ is then $27 \%$ and $31 \%$ for the VITAL method and the actuated method correspondingly. The results also indicate that both traffic dependent control methods have the similar reduction in $\mathrm{NO}_{\mathrm{x}}$ and $\mathrm{PM}_{\mathrm{x}}$, when the traffic flow is quite low, e.g. between 11 and 15 o'clock. When the traffic flow increases and approaches the amount of traffic during the peak hour period, the VITAL method performs better than the actuated method. 


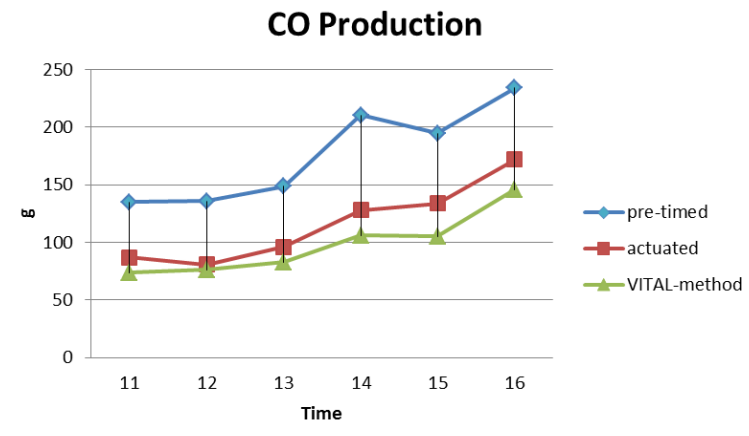

(a) Hourly CO productions of three different control methods

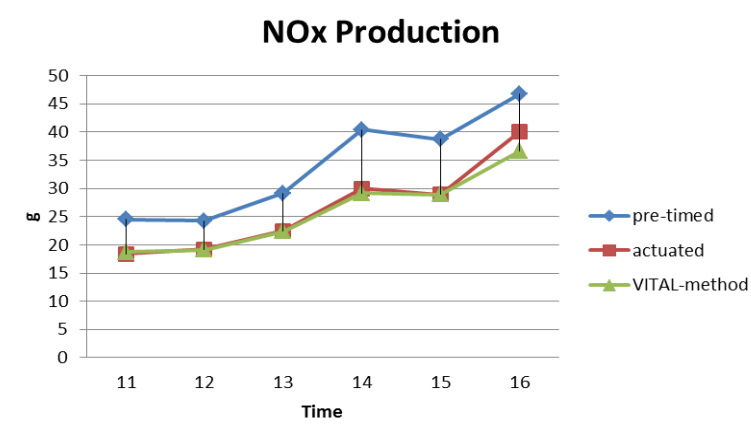

(b) Hourly $\mathrm{NO}_{x}$ productions of three different control methods

PMx Production

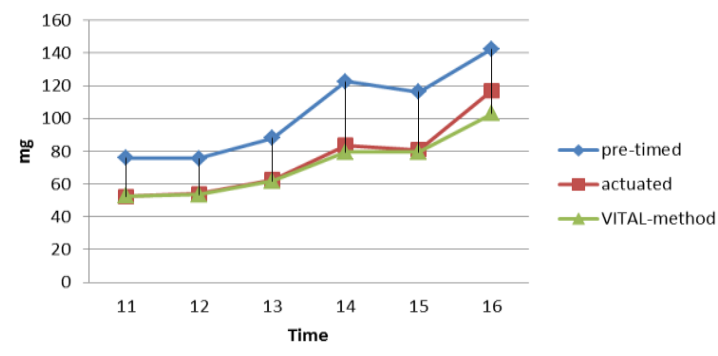

(c) Hourly $\mathrm{PM}_{\mathrm{x}}$ productions of three different control methods

Figure 8. Comparion of the simulated emissions.

\section{B. Actual results}

The survey of the intersection operation efficiency has been conducted with the point sampling method during the field test. The vehicular queuing times at the intersection are collected. The stopping vehicles in the front of the waiting line and the pass-through vehicles on each approach are counted every 15 seconds. The sum of these two types of vehicles is the number of the total vehicles travelling on the respective approach. If one vehicle has stopped more than 15 seconds, it is counted as a stopped vehicle every 15 seconds. In addition, it is ensured that the cycle length cannot be divisible by the selected counting interval (15 seconds). According to the collected data, the delay time and the vehicle stopping rate on each approach are calculated with use of the formulae shown in (1), (2) and (3).

$$
\begin{gathered}
\kappa_{i}=v_{s, i} \times 15 \\
\mu_{i}=\kappa_{i} / v_{i}
\end{gathered}
$$

$$
\lambda_{i}=v_{s, i} / v_{i}
$$

where $\kappa_{i}$ : total delay time; $v_{s, i}$ : the number of the stopped vehicles on the approach $i ; \mu_{i}$ : average delay time on approach $\mathrm{i} ; v_{i}$ : the number of the total vehicles on approach i; $\lambda_{i}$ : vehicle stopping rate on the approach $i$.

The average delay and the vehicle stopping rate at the subject intersection are 45 seconds and $64 \%$ before the proposed model is applied. After the model application the values has become 35 seconds and 66\% correspondingly. The Reduction in the traffic delay is $21 \%$. The vehicle stopping rate has however slightly increased $3 \%$ after the model application. It is mainly since the signal phases are switched frequently for better allocating the available green times. More vehicles may therefore need to stop, but shortly.

Fig. 9 shows the result comparison for each driving direction. The proposed model has reduced the average delay at most directions except the southbound direction (35 seconds with the pre-timed control and 38 seconds with the VITAL method). It is mainly due to that the simulated objects and the real vehicles cannot perfectly synchronize with each other yet. Sometimes, some simulated vehicles are already left the intersection and the signal phase is then switched to red. However, the respective vehicles in practice are still in front of the intersection and therefore need to wait for the green time. Fig. 9 (b) indicates that the eastbound traffic has around $20 \%$ less vehicle stopping rate after applying the proposed model. There are higher vehicle stopping rate at the other three directions, especially at the southbound direction. It is also due to the frequent phase switching. Moreover, the left turning traffic at the southbound direction is higher than the through traffic, and needs more time to cross the intersection in practice than in the simulation. Thus, less left turning traffic can pass the intersection and more stops need to be made. 


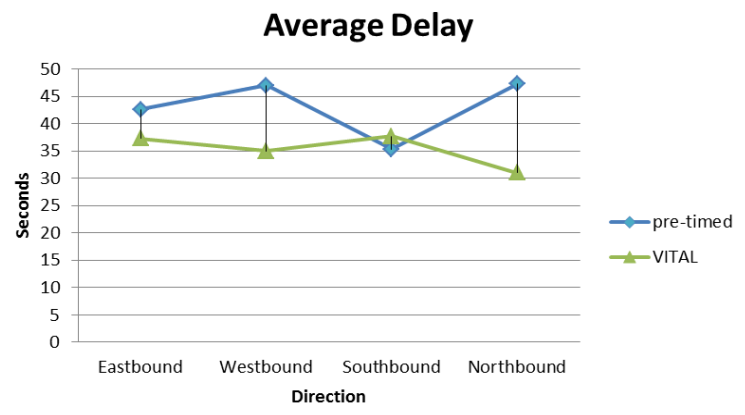

(a) Average delays

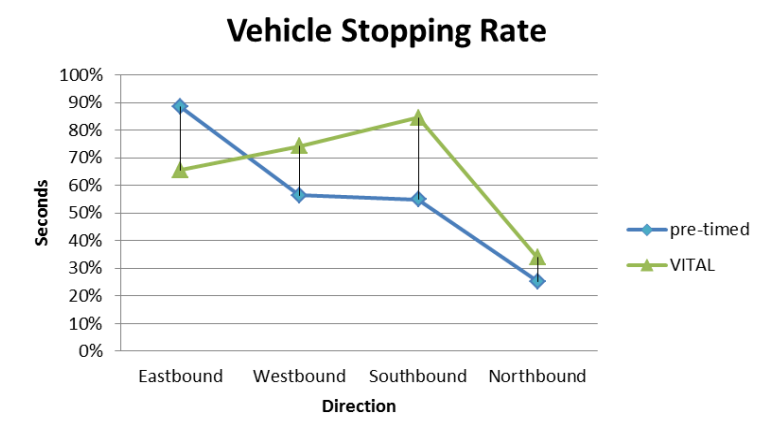

(b) Vehicle stopping rate

Figure 9. Intersection performances before and after applying the online traffic simulation and optimization model.

\section{CONCLUSION}

In this study, a real-time traffic simulation and signal optimization model is proposed and implemented on a small IPC with high applicability and practicability. This IPC serves as the interface between the traffic signal controller and the video traffic detectors. Given the ICT data and the current traffic signal programs, only the maxi- und minimum green time durations for each phase need to be set. This study further demonstrates that, with use of the ICT data, the varied traffic states can be easily captured in real time and the intersection's operation efficiency can be significantly improved with the proposed model in practice.

The results both from the simulation study and the real field test show that the proposed model can significantly improve the operation efficiency, i.e. less stopping duration, less traffic delays and less emission productions at the subject intersection. Due to the illegal crossing behavior by pedestrians and some "imperfect" driving behaviors in practice the achieved improvement in the real field test is less than that in the simulation study. The overall performance of the proposed model is however still better than the pre-timed and the actuated signal control methods. Some enhancements on the synchronization of the simulated and the real objects and on the adjustment of the crossing time for left turning traffic will be undertaken in order to further improve the model performance. For further testing and developing the proposed model it is planned to firstly extend the field test area to a road corridor with several intersections and then to a complete traffic network.

\section{ACKNOWLEDGMENT}

This work is carried out within the project OptimUM (Optimization of Urban Traffic Management towards Environment Friendly and Safe Mobility) which is financially supported by the Helmholtz CAS joint research groups (HCJRG) of the Helmholtz association of German Research Centers. This support is gratefully acknowledged.

\section{REFERENCES}

[1] Y. W. Xiao, X. F. Guo, and H. X. Huang, "Design of Selfadaptive Intelligent Traffic Signal Machine System," Computer Engineering, vol. 35, issue 7, pp. 220-222, 2009.

[2] Q. Liu, "Study on platoon-based Urban Adaptive Traffic Control Method," MS. thesis, Dalian University of Technology, Dalian, ON, China, 2008.

[3] L. J. Li, "Research on Intelligent Optimization Algorithm and Its Applications," MS. thesis, Jiangnan University, Wuxi, ON, China, 2011.

[4] H. Ding, W .H. Zhang, and X. Y. Zheng, "Multi-state Flow Signal Control Based on Traffic Prediction," China Journal of Highway \& Transport, vol. 25, issue 5, pp. 126-133, 2012.

[5] X. L. Bai, "Based on SCATS System signal control scheme design and optimization," MS. thesis, North China University of Technology, Beijing, ON, China, 2011.

[6] L. Y. Sui, W. Li, and J. J. Shi, "The Analysis and Application Actuality of Traffic Signal Control System - SCOOT and ACTRA in Beijing," Road Traffic \& Safety, issue 2, pp. 1013, 2007.

[7] G. W. Zhang, "The Application and Development of Dynamic Traffic Simulation Theory," Journal of Transportation Systems Engineering \& Information Technology, vol. 14, issue 2, pp. 1-6, 2014.

[8] P. Q. Lin, J. H. Liu, and K. B. Yao, "Microscopic Online Simulation Method of Road Traffic Accident Emergency Disposal," Journal of System Simulation, vol. 28, issue 5, pp. 1158-1164, 2016.

[9] W. Wu, B. Y. Shi, and J. Xie, "Parameter calibration in realtime traffic control microsimulation," Journal of Tongji University, vol. 39, issue 6, pp. 842-847, 2011.

[10] Y. Z. Zhang, Y. Y. Zheng, and Z. X. Li, "Online Simulation System of Urban Traffic Control," Communications Standardization, issue 9, pp. 87-89, 2008.

[11] C. X. Deng, H. T. Li, "Research and Design of Data Driven Intelligent Traffic Virtual Simulation System," Computer Knowledge and Technology, vol. 9, issue 20, pp. 4689-4691, 2013.

[12] Y. Jia, J. P. Wu, and Y. M. Du, "Study on FLOWSIM and its Application for Isolated Signalized Intersection Assessment," Journal of System Simulation, vol. 26, issue 10, pp. 25292534, 2014.

[13] R. Oertel and P. Wagner, "Delay-time actuated traffic signal control for an isolated inter-section," Proc. of the 90th Annual Meeting Transportation Research Board (TRB), Jan. 2011.

[14] Transportation Research Board, HCM - Highway Capacity Manual, Washington, D.C.: Transportation Research Board, 2010.

[15] R. Oertel, J. Erdmann, A. Mann and P. Wagner, "VITAL Vehicle-Actuated Intelligent Traffic Signal Control," 
Helmholtz Innovation Days 2013 - Partnering Research and Business, 2013.

[16] R. Oertel, J. Erdmann, A. Mann, J. Trumpold and P. Wagner, "VITAL - Vehicle-Actuated Intelligent Traffic Signal Control: Validation of two new Control Approaches in the Field," Proc. of the 11th ITS European Congress, June 2016.

[17] Y.-P. Flötteröd, J. Trumpold, A. Sohr, H. Saul, F. Wu, Y. Shi, „Monitoring and Reducing Vehicular Impact on Air Quality at Intersections - A Real Case Study in China," Proc. of the 17th International Conference on Urban Transport and the Environment, Rom, Italy, Sep. 2017.

[18] D. Krajzewicz, J. Erdmann, M. Behrisch and L. Bieker, „Recent development and applications of SUMO - Simulation of Urban Mobility," International Journal On Advances in Systems and Measurements, 5(3\&4), Dec. 2012, pp-128-138.
[19] SUMO, Simulation of Urban MObility - Wiki website, http://sumo.dlr.de/wiki/, accessed on 10 July 2017.

[20] Y.-P. Flötteröd, "SUMO-Cadyts calibration with limited data quality," Proc. of the SUMO User Conference - Towards Simulation for Autonomous Mobility, May 2017, pp. 155162.

[21] HABEFA, http://www.hbefa.net/e/index.html, accessed on 5 Oct. 2017.

[22] SUMO, SUMO - Simulation/Traffic Lights website, http://sumo.dlr.de/wiki/Simulation/Traffic_Lights, accessed on 5 October 2017. 
Please fill in the Authors' background:

\begin{tabular}{|c|c|c|c|c|}
\hline \multicolumn{5}{|c|}{$\begin{array}{l}\text { Position can be chosen from: } \\
\text { Prof. / Assoc. Prof. / Asst. Prof. / Lect. / Dr. / Ph. D Candidate / Postgraduate / Ms. }\end{array}$} \\
\hline Full Name & Email address & Position & $\begin{array}{l}\text { Research } \\
\text { Interests }\end{array}$ & $\begin{array}{l}\text { Personal website } \\
\text { (if any) }\end{array}$ \\
\hline Zijun Liang & 953609319@qq.com, & Ph.D. Candidate & $\begin{array}{l}\text { Transportation } \\
\text { planning theories } \\
\text { and practices }\end{array}$ & \\
\hline Hong Chen & glch@chd.edu.cn & Prof. & $\begin{array}{l}\text { Transportation } \\
\text { planning and } \\
\text { management }\end{array}$ & \\
\hline $\begin{array}{l}\text { Yun-Pang } \\
\text { Flötteröd }\end{array}$ & $\begin{array}{l}\text { yun- } \\
\text { pang.floetteroed@dlr.de }\end{array}$ & Dr.-Ing. & $\begin{array}{l}\text { Traffic demand } \\
\text { and assignment } \\
\text { modelling, traffic } \\
\text { simulation }\end{array}$ & \\
\hline Alexander Sohr & alexander.sohr@dlr.de & Dipl.-Ing. & $\begin{array}{l}\text { Data } \\
\text { management, } \\
\text { data fusion }\end{array}$ & \\
\hline Xiaoxu Bei & xiaoxu.bei@dlr.de & Dipl.-Ing. & $\begin{array}{l}\text { Traffic } \\
\text { management }\end{array}$ & \\
\hline $\begin{array}{l}\text { Maximiliano } \\
\text { Bottazzi }\end{array}$ & Maximiliano.Bottazzi@dlr.de & Ms. & $\begin{array}{l}\text { Data } \\
\text { management, } \\
\text { data fusion }\end{array}$ & \\
\hline Jan Trumpold & Jan.Trumpold@dlr.de & Dipl.-Ing. & $\begin{array}{l}\text { System } \\
\text { development }\end{array}$ & \\
\hline
\end{tabular}

*This form helps us to understand your paper better; the form itself will not be published. 